\title{
CORRELATION-BASED MODEL FOR EVALUATING GROUND PENETRATING RADAR (GPR) DATA OF CONCRETE BRIDGE DECKS
}

\author{
*K. Dinh \\ Graduate Student, Concordia University \\ 1515 Sainte-Catherine $W$. \\ Montreal, Canada H3G 1 M7 \\ (*Corresponding author: dinhkien_huce@yahoo.com) \\ T. Zayed \\ Associate Professor, Concordia University \\ 1515 Sainte-Catherine W. \\ Montreal, Canada H3G 1 M7
}




\title{
CORRELATION-BASED MODEL FOR EVALUATING GROUND PENETRATING RADAR (GPR) DATA OF CONCRETE BRIDGE DECKS
}

\begin{abstract}
The Ground Penetrating Radar (GPR) has been studied for a long time as a non-destructive evaluation (NDE) technology for inspection of concrete structures. Currently, the most widely used technique for interpreting GPR data of concrete bridge decks is the one that based on the amplitudes measured at various interfaces such as asphalt-concrete, top rebar, or slab bottom. The assumption behind this technique is that a sound concrete deck should have the same reflection amplitude at these interfaces while any low number would indicate some sign of deterioration. Unfortunately, this assumption is not completely valid in most cases. As a consequence, the reported test results usually do not reflect real condition of concrete bridge decks in question. The main goal of this paper is therefore twofold: (1) to discuss the limitations of the amplitude analysis method, and (2) to propose a new model that interprets GPR data of concrete bridge decks. The model methodology is based on the comparison of GPR A-scans between two inspections, using the so-called correlation analysis. The results, indicated by the correlation coefficients, are then employed to develop a contour map that estimates different levels of deterioration. The model is then implemented to a case study in order to illustrate its methodology.
\end{abstract}

\section{KEYWORDS}

Ground Penetrating Radar (GPR), Non-destructive Evaluation (NDE), Concrete Structures, Concrete Bridge Decks, and Bridge Inspection.

\section{INTRODUCTION}

Inspection and condition assessment of bridge structures provide necessary inputs for planning bridge rehabilitation and maintenance activities. Among various elements, concrete bridge decks have always been the focus of transportation agencies in both Canada and the United States because of high deterioration rate and high upfront cost associated with their repair and rehabilitation. Although the deterioration of concrete is a result of combined effects of many complex phenomena, deterioration of most concrete bridge decks in North America is associated with corrosion of reinforcing bars that caused by de-icing salt applied on bridge decks during winter or by salt in seawater for bridges built in marine environments (Qian 2004).

According to Carino (2004), although the exact mechanism is not known, it is observed that when the presence of chloride reaches a certain extent, it breaks down the passive oxide film and the condition is ready for corrosion to be initiated. The reinforcement corrosion then happens because many tiny electrolytic cells are formed. In these cells, the water in the pores of the paste contains various dissolved ions and serves as the electrolyte while heterogeneities in the surface of the steel cause some regions of the bars to act as the anodes and other regions to act as the cathodes. Since the products of corrosion occupy much bigger volume than the original steel, they produce internal stresses and causes delamination in concrete structure. At the beginning, the delaminations usually have a small size and locate separately. Once growing up and encountering one another, they become a spall that can be visually observed.

Because visual assessment can only detect crack or spall when concrete deterioration has matured, non-destructive evaluation (NDE) has been studied by bridge community as an alternative for concrete bridge inspection. Among various NDE technologies, the Ground Penetrating Radar (GPR) has attracted an extensive number of researches. This technology can collect data with high speed and precision while theoretically it can detect most common defects in concrete structures such as corrosion or delamination. 
Unfortunately, GPR is still of limited practice due to the limitation of available data evaluation, namely amplitude analysis method.

\section{RESEARCH OBJECTIVE}

The ultimate goal of this study is to develop an alternative approach for analyzing GPR data of concrete bridge deck. In order to achieve that goal, the research needs to obtain the following subobjectives for concrete bridge decks:

(i) Study GPR as a nondestructive evaluation technology.

(ii) Identify limitations of amplitude data analysis method.

(iii) Develop a model for interpreting and mapping GPR data.

\section{BACKGROUND}

\section{Ground Penetrating Radar (GPR)}

Ground Penetrating Radar (GPR) is a non-destructive technique that has been successfully used in many civil engineering applications such as for detecting cavities below airfield pavements, determining concrete thickness, locating voids or reinforcing bars, and identifying concrete deterioration. The fundamental working principle of radar is based on the propagation behavior of electromagnetic (EM) waves in which when a beam of EM energy encounters an interface between two media of different dielectric constants, a portion of energy is reflected back while the remainder penetrates through the interface into the second medium.

To inspect horizontal structures such as pavement or bridge deck, an antenna is dragged manually over the inspected surface or attached to a vehicle in order to scan with traffic speed. This antenna transmits a short pulse of electromagnetic energy into the surveyed structure, commonly ranging from 1 to 3 nanoseconds (ns) in duration (ACI 288.2R-98). The energy reflected at various material interfaces is then received by another antenna (bistatic mode), or sometimes by the same antenna (monostatic mode), to produce the output signal that is proportional to the amplitude of the reflected electromagnetic field. This process is repeated at a certain pulse repetition frequency, typically greater than $100 \mathrm{kHz}$, as the antenna is moved along the survey path. The result is then presented as an individual waveform (A-scan) or a GPR image (B-scan) as shown in Fig. 1.

\section{AMPLITUDE ANALYSIS OF GPR DATA}

Regarding data analysis and interpretation, currently, the most commonly used technique for mapping the information derived from GPR data is the method that based on reflection amplitudes. Fundamentally, this technique makes use of signal processing to compute the amplitudes and arrival times of significant reflection peaks in the radar waveform (ACI 228.2R-98). These values are then used to calculate and display structural properties as a depth profile or a contour map. Detailed description and discussion of this method is followed.

As known from theory, the two main factors affecting the propagation and reflection of electromagnetic waves are the dielectric constant and electrical conductivity of the media being probed. The corrosion areas in reinforced concrete decks are usually associated with high moisture and chloride content (Maser \& Bernhardt, 2000). The presence of these substances affects the dielectric constant and electrical conductivity of concrete. These parameters, in turn, have an impact on the amplitude of asphaltconcrete interface reflection. Therefore for overlaid concrete bridge deck, by investigating the variation of reflection amplitudes in overlay-concrete interface, corrosion-induced damage areas on the deck can be inferred or detected (Maser \& Bernhardt, 2000). 


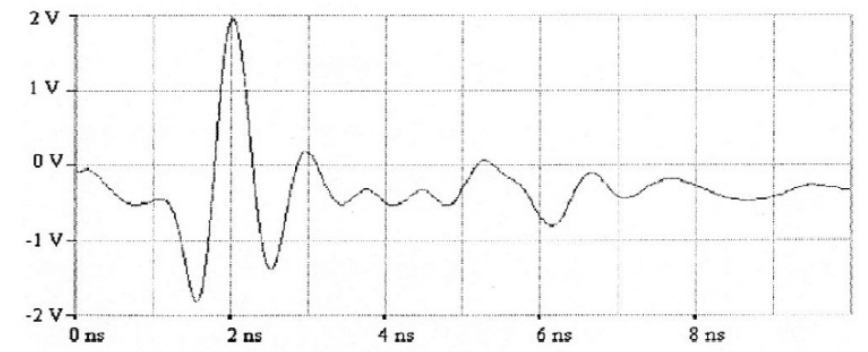

(a) A-scan

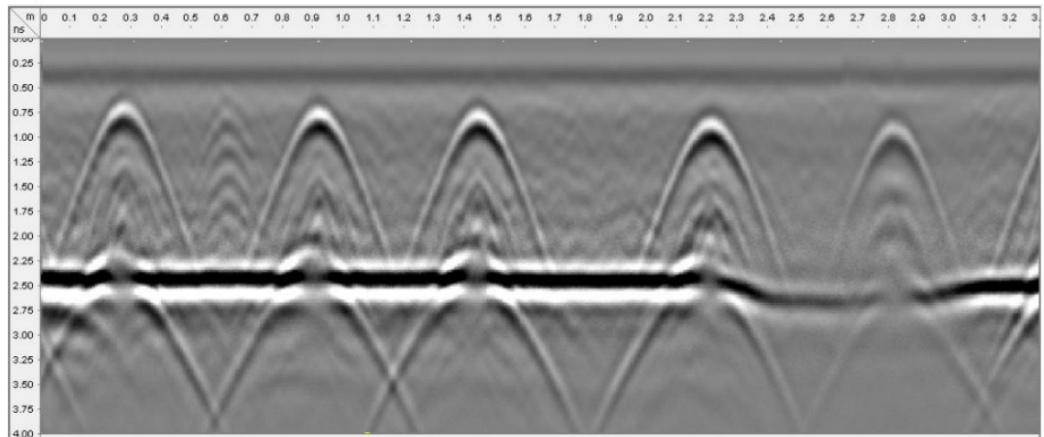

(b) B-scan

Figure 1 Typical GPR output displays

In case of the bare concrete bridge decks, the concrete surface tends to dry out and the moisture related variations are not necessarily observed. As an alternative, the attenuation of the deck bottom or top rebar reflections is used to detect concrete deterioration (ASTM D 6087; Maser \& Bernhardt 2000). The measurements for all scans are then used to create a contour map of reflection amplitudes. Locations of deteriorated concrete correspond to the areas where the reflection amplitudes are lower than a predetermined threshold value (ASTM D 6087).

According to Tarussov et al. (2013), applied projects have revealed that neither the ASTM standard nor numerical analysis in general consistently provide the expected results. They pointed out several reasons that can explain this inefficiency. Specifically, they found that several factors can affect the reflection amplitudes such as reinforcing bar depth variation, spacing, surface anomalies, structural formation, use of horn antenna, interference and so on. So far, variation in reinforcing bar depth is the only factor that has been considered by researchers for amplitude correction (Barnes et al., 2008).

\section{RESEARCH METHODOLOGY AND MODEL DEVELOPMENT}

It is recognized in this study that the appropriate way to find defects associated with concrete bridge deterioration is by comparing current GPR signals with themselves, i.e. at the same location, but taken previously when the bridge is newly constructed. Specifically, GPR data along with their exact locations, i.e. scanning paths, can be recorded and stored in the database right after the concrete bridge deck is constructed and the concrete is completely cured. These data are termed in this paper as "As-Built" GPR data. Periodically, each time during operation and maintenance stage when the deck is inspected, new GPR data, or "As-Is" data, at exactly the same location will be collected. Then, the comparison for each pair of GPR signals, "As-Is" versus "As-Built", collected at the same location will be performed using the correlation-based model developed in this study. Based on the comparison result, condition at the inspected location will be predicted. Theoretically, it is clear that the more similarity between the two signals, the better quality of the concrete is, at the inspected location. 


\section{Normalized Cross-Correlation (NCC) Technique}

Normalized Cross-Correlation (NCC), a signal processing technique, has been found to be an excellent mathematical tool to bring the above idea into reality. This technique is known in signal processing as a tool to measure the similarity between two signals as a function of a time-lag applied to one of them. According to Tsai and Lin (2003), correlation-based methods have been used extensively in many applications such as object recognition, motion analysis, industrial inspection, and so on. For example, Tsai et al. (2003) studied the use of NCC for defect detection in complicated images of industrial inspection. Giachetti (2000) proposed using pattern (template) matching to compute image motion from a sequence of two or more images in which the displacement between two images is calculated based on the correlation measure between them. In a very interesting study, Brunelli \& Poggio (1993) compared two different techniques for human face recognition in which the first technique was based on the computation of a set of geometrical features while the second one was based on correlation-based template matching. The same database that included frontal images of 47 people was used for the two techniques. The result favored template matching in which it got perfect recognition while the method based on geometrical features obtained only 90 percent correct recognition.

Regarding the computation algorithm, the cross-correlation function between the signals $x(t)$ and $y(t)$ is defined as a convolution between two signals as shown in Equation 1 (Mahafza, 2000). It is noted in the equation that $x^{*}$ denotes the complex conjugate of $x$. The peak value of the obtained cross correlation function $R_{x y}(t)$ indicates how good the correlation is.

$$
R_{x y}(\tau)=\int_{-\infty}^{+\infty} x^{*}(t) y(t+\tau) d t
$$

Where:

$t=$ time

$\tau=$ time lag between the two signals

If the signals $x(t)$ and $y(t)$ are discrete resulting from digitization, the cross correlation function between them is defined as shown in Equation 2.

$$
R_{x y}(m)=\sum_{n=-\infty}^{+\infty} x^{*}(n) y(n+m)
$$

Where:

$n=$ discrete time resulting from digitization

$m=$ discrete time lag between the two signals

To easily compare the similarity between two signals, the normalized cross correlation (NCC) is commonly used. Basically, the normalized cross correlation function $\rho_{x y}$ between two one-dimensional signals $x(t)$ and $y(t)$ is the normalized cross covariance function calculated as shown in Equation 3. As can be seen, the value of $\rho_{x y}$ lies between -1 and 1 in which the closer to unity, the more similarity between the two signals.

$$
\rho_{x y}(\tau)=\frac{\gamma_{x y}(\tau)}{\sigma_{x} \sigma_{y}}
$$

Where:

$\tau=$ time lag between the two signals

$\gamma_{x y}(\tau)=E\left[\left(x_{t}-\mu_{x}\right)\left(y_{t+\tau}-\mu_{y}\right)\right]$

$\mu_{x}$ and $\mu_{y}=$ means of $x_{t}$ and $y_{t}$, respectively

$\sigma_{x}$ and $\sigma_{y}=$ standard deviations of $x_{t}$ and $y_{t}$, respectively 
Fig. 2 illustrates the above idea of using NCC for comparing the similarity between two GPR waveforms. Each waveform, Fig. 2a and b, is sampled and the voltage amplitudes are measured at 512 points along each scan. The correlation computation and graphical drawing were performed using MATLAB for these two waveforms. It can be visualized that firstly one waveform is slid over the other and then for each sliding position, the correlation coefficient is computed for the two signals. The result of NCC computation is shown in Fig. 2c with a total of 1023, i.e., 2x512-1, sliding positions. As can be seen, the position with the highest correlation coefficient of 0.8588 is at point 512 , corresponding to when the time lag $(\tau)$ between two signals is zero.

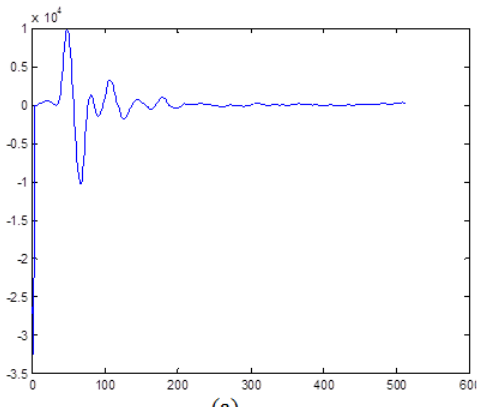

(a)
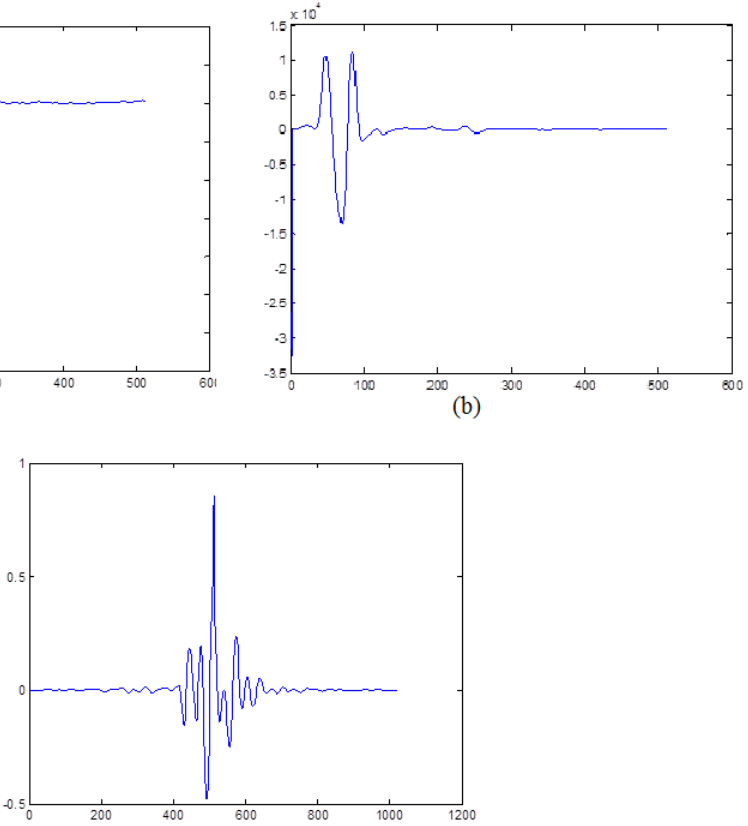

(c)

Figure 2 Normalized cross correlation between two GPR signals

\section{Model for Interpreting and mapping GPR data of concrete bridge decks}

For the proposed model in this study, the NCC technique is used and the correlation coefficient is computed with the time lag between two GPR signals $(\tau)$ being zero. It is suggested that when a concrete bridge deck is newly constructed, GPR is used to scan the deck and the data (As-Built) is stored. Since the GPR data is taken with the new deck, it can be assumed that all those GPR signals are associated with sound concrete condition. For the next time, several years later, when the deck is inspected, the same GPR equipment is used and GPR signals (As-Is) are taken at the same location as the original ones. For each location, the correlation coefficient between two signals, As-Is versus As-Built, is calculated using Equation 5 with the time lag between two GPR signals $(\tau)$ being zero. Thus, for any inspected location on the deck, only one correlation coefficient is obtained. Then, the correlation coefficients for these discrete locations are used to develop the coefficients for the whole deck using interpolation algorithm - an approximation method. By using pre-determined thresholds for these coefficients, delamination and corrosion regions can be specified or estimated. The proposed model is illustrated in Fig. 3.

Regarding the exact values of the pre-determined thresholds mentioned above, they should be calibrated by statistical studies with ground-truth information. However, due to the unavailability of such data at the current stage of this study, in order to illustrate the idea, this paper assumes the thresholds of correlation coefficient for corrosion and delamination are 0.95 and 0.90 , respectively. The reason for the greater value of the assumed threshold with corrosion in comparison to delamination is that when a 
delamination appears in concrete, it will become an interface for reflection of GPR energy and distort the shape of reflected GPR signals; while the corrosion only attenuates the amplitude of GPR signals, resulting in lower correlation coefficient.

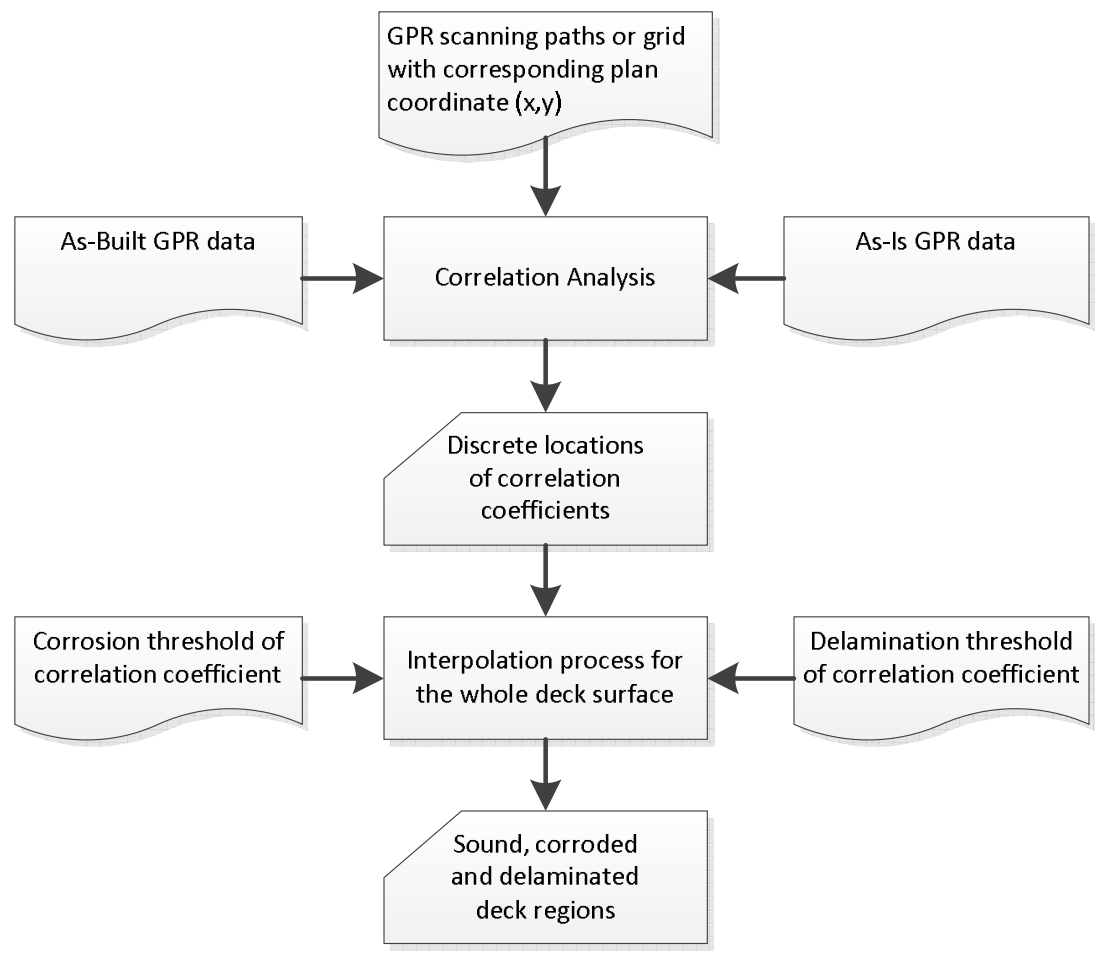

Figure 3 Correlation-based concrete deck condition assessment model

\section{IMPLEMENTATION TO AN ILLUSTRATIVE EXAMPLE}

This section explains in detail the proposed model using an illustrated example. Specifically, Fig. 4 shows the plan-view of an exemplary concrete bridge deck in which the locations of GPR scanning paths are clearly specified and indicated. As can be seen, each scan line, with scan direction, is named on the plan, for example F77, in order to differentiate it with others. For illustrative purpose, the proposed model is implemented for the portion of this example deck corresponding to the scanning paths from F137 to F151. It is noted that while the GPR data is taken from a real concrete bridge deck; the original signals, i.e. taken when the deck was newly constructed, are assumed. Fig. 5 shows the sections of two GPR profiles for performing the correlation analysis in which Fig. 5a is the profile section of scanning path F137 taken recently while Fig. $5 \mathrm{~b}$ is the same profile section assumed to be taken when the deck was newly constructed. Each profile contains the information of many scans. For instance, the profile for scanning path F137 consists of 6868 individual signals. The correlation analysis is then implemented in MATLAB, using equation (5) with the time lag $(\tau)$ of zero, for each pair of the signals at the same location on the two profiles. The obtained result is the correlation coefficient, lying between minus unity and unity.

As previously explained, the closer to unity the correlation coefficient is, the more similar between two compared waveforms. In other words, the lower correlation coefficient, the more deteriorated the concrete is at the location where the two signals are taken. Thus, if two assumed thresholds (i.e. 0.95 and 0.90 ) are used, the concrete condition can be predicted, given the value of correlation coefficient at that location. For example, if the correlation coefficient computed at a location is 0.97 , it can be expected that the concrete is good at that location, since 0.97 is greater than 0.95 (corrosion coefficient threshold). Based 
on the same logic, if a location has the correlation coefficient of 0.92 , corrosion condition can be predicted for concrete at that location.

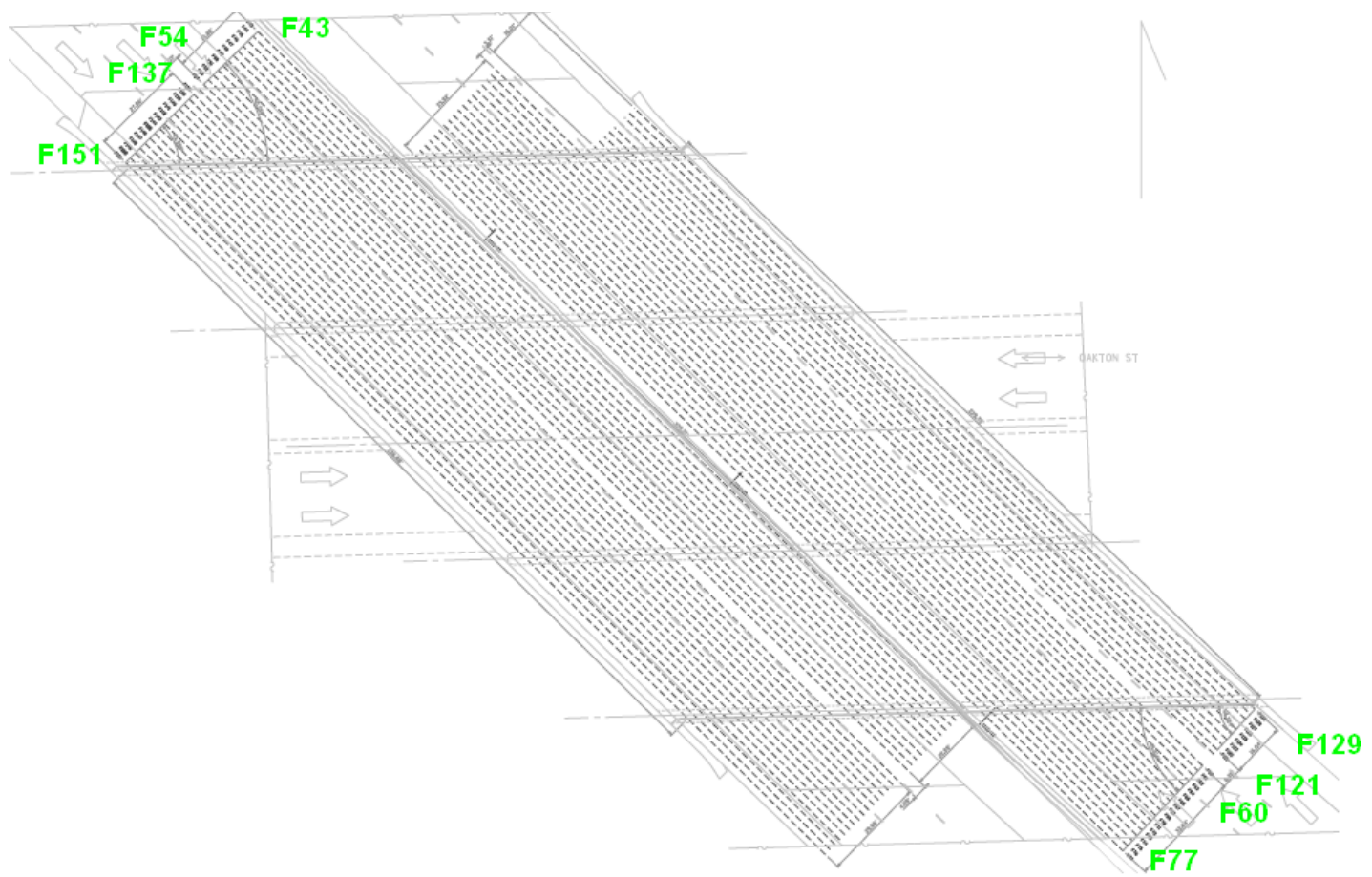

Figure 4 Bridge deck plan-view with locations of GPR scanning paths

Because scanned positions, with $\mathrm{x}$ and $\mathrm{y}$ coordinates, represent discrete locations on the bridge deck surface, it is needed to predict the condition for the whole bridge deck area. This is performed in AutoCAD Map 3D, an Autodesk software package. Finally, a contour map coded in different colors is generated with two pre-determined thresholds, 0.95 and 0.90 , as assumed above. The result obtained for the section of the example deck corresponding to the scanning paths from F137 to F151, with unit in feet, is shown in Fig. 6. In the figure, green color is used to indicate sound concrete (i.e. correlation coefficient is greater than 0.95), while the locations associated with delamination (i.e. correlation is below 0.90) and corrosions (i.e. correlation is between 0.90 and 0.95 ) are represented by red and yellow colors, respectively.

\section{DISCUSSION}

The illustrative example above clearly shows the simplicity of the proposed model. A big potential of this method is that it can be extended for implementation on the entire bridge 3D model. It can be imagined that the final output in such case would be a bridge 3D model showing different structural defects, coded in different colors, as well as their exact locations. This task is obviously feasible when 3D scanning technology is now available for the bridge 3D model to be easily constructed.

Unlike the traditional numerical analysis, correlation analysis can eliminate all anomalies due to rebar depth variation or because of structural formation. As explained before, the numerical analysis cannot differentiate the attenuated amplitude due to deterioration with the one originated from rebar depth variation or structural formation. Thus, correlation-based method can filter all defects associated with the deterioration process such as corrosion and delamination.

Since one important requirement for the approach to work is the need to properly align the GPR scans acquired at different times, one may argue that inaccurate alignment would lead to wrong analysis and inappropriate decisions. Regarding this issue, a small test has been set up in which the same test line in 
a concrete parking garage was scanned twice, minutes apart in time, by the GSSI SIR-3000 equipment. The deviation was recorded up to $3 \mathrm{~mm}$ and each line contained 496 individual scans. The correlation analysis was then performed. The result shown that the lowest correlation coefficient between these two profiles was very high, i.e., 0.998087 . This means that the required positioning for the proposed model can be obtained.

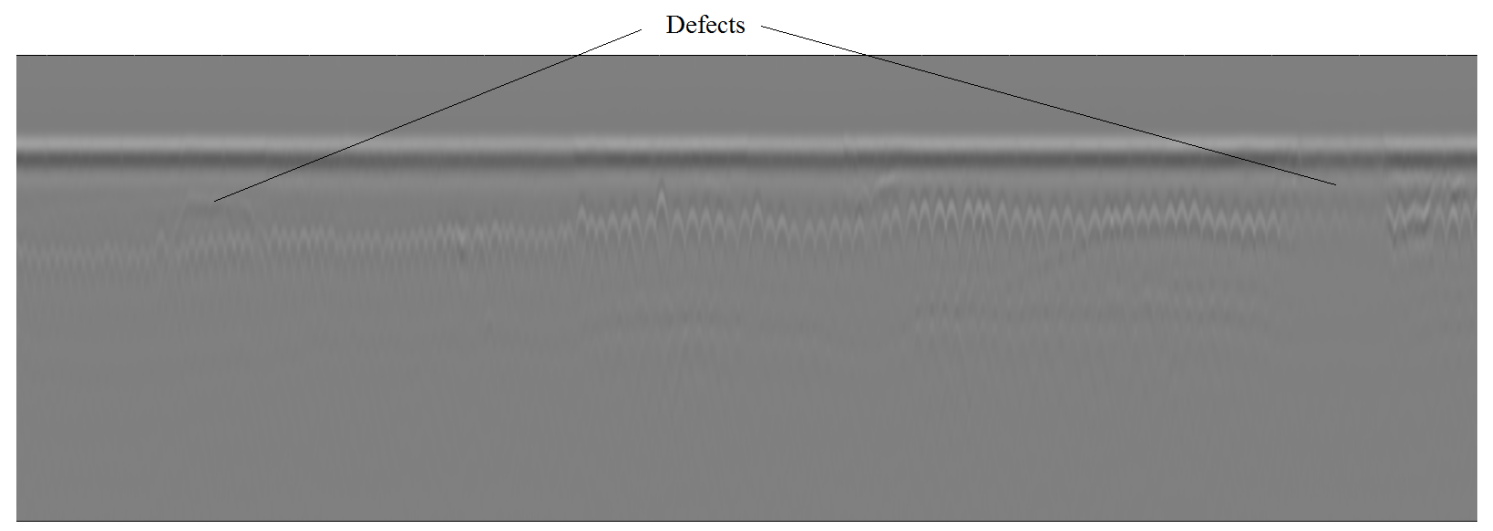

(a) Current GPR protile

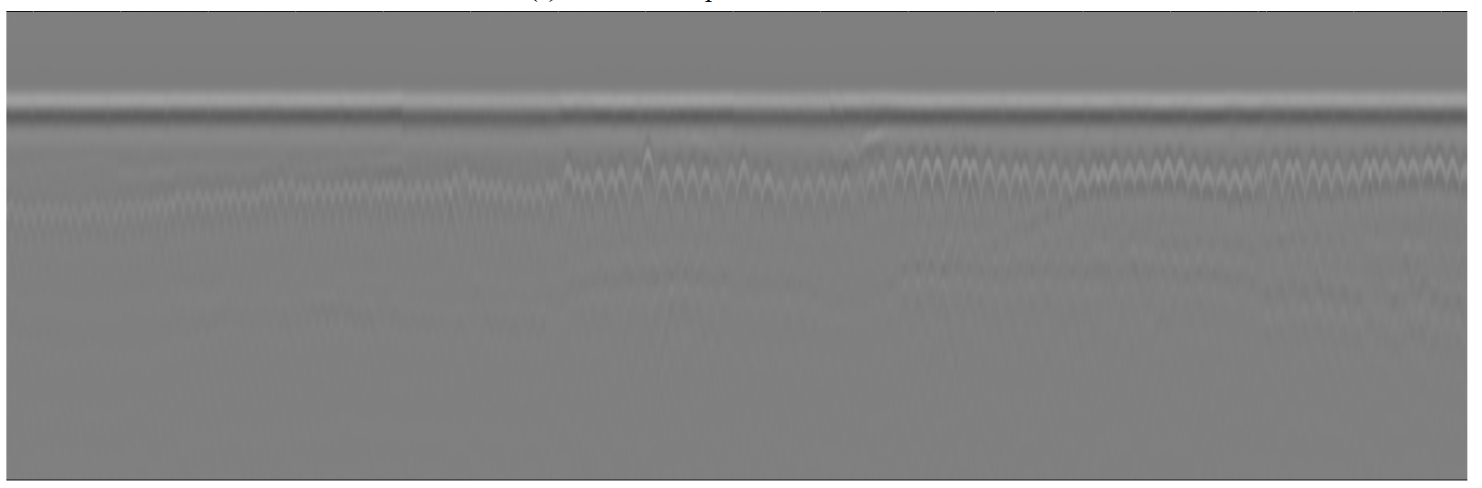

(b) Original GPR profile

Figure 5 Current and assumed original GPR profiles

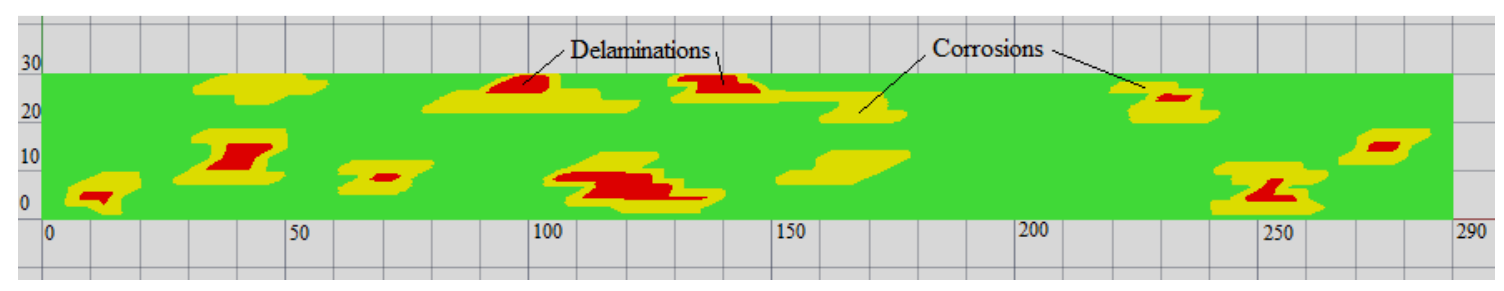

Figure 6 Deteriorated map provided by the proposed model

As can be seen, the basic idea behind the method is easy to understand with the assumption that the concrete is sound when the decks are newly constructed. The only drawback of the proposed method compared with visual analysis is that it requires the original signals for implementation. Obviously, this will result in more inspection cost in the short-term associated with the first data collection. However, it is 
argued that this cost will be offset and paid back many times its initial value, because of reduced inspection costs later on.

\section{CONCLUSION}

Transportation agencies need accurate inspection techniques for assessing condition of their bridges, especially concrete bridge decks. GPR has been found to be one of the most appropriate nondestructive evaluation techniques for that purpose. Because amplitude analysis ignores most of the information contained in GPR profiles and leads to errors, the model using correlation analysis technique proposed in this paper can solve the problem of that data analysis method. At one hand, it can eliminate anomalies unrelated to deterioration. On the other hand, implementation in the case example has shown that it can be executed in a computer with the possibility of automation and applicable to the entire bridge with the 3D model. The output of the proposed methodology is a 2D map or 3D model that clearly shows the location or area of hidden defects. The only drawback of the model is that it requires original data collected when the decks are newly constructed. However, it is believed that the long-term benefits will outweigh the upfront cost of this first data collection. Finally, it is expected that the proposed model will be

further developed and validated in an on-going research project when statistically significant data has been collected.

\section{REFERENCES}

ACI 228.2R-98 (2004). Nondestructive Test Methods for Evaluation of Concrete in Structures. American Concrete Institute.

ASTM D6087 - 08 (2010). Standard Test Method for Evaluating Asphalt-Covered Concrete Bridge Decks Using Ground Penetrating Radar. Annual Book of ASTM Standards, American Society for Testing and Materials, Vol. 04.03.

Barnes, C., Trottier, J. F., \& Forgeron, D. (2008). Improved Concrete Bridge Deck Evaluation Using GPR by Accounting for Signal Depth-Amplitude Effects. NDT \& E International, Vol. 41, No. 6, September, 427-433.

Brunelli, R., \& Poggio, T. (1993). Face Recognition: Features versus Templates. IEEE Transactions on Pattern Analysis and Machine Intelligence, Vol 15, No. 10, October, 1042-1052.

Carino, N.J. (2004). Method to Evaluate Corrosion of Reinforcement. . In Malhotra,V.M and Carino, N.J., (Eds.), Handbook on Nondestructive Testing of Concrete, 2nd Edition, ASTM \& CRC

Giachetti, A. (2000). Matching techniques to compute image motion. Image and Vision Computing, Elsevier, Vol. 18, Iss. 3, February, 247-260.

Mahafza, B.R. (2000). Radar Systems Analysis and Design Using MATLAB. Chapman \& Hall/CRC.

Maser, K., \& Bernhardt, M. (2000). Statewide Bridge Deck Survey Using Ground Penetrating Radar. Structural Materials Technology IV - An NDT Conference, Atlantic, New Jersey, 31-37.

Qian, S. (2004). Preventing Rebar Corrosion in Concrete Structures. Retrieved from National Research Coucil Canada website: www.nrc-cnrc.gc.ca/obj/irc/doc/pubs/nrcc47625/nrcc47625.pdf

Tarussov, A., Vandry, M., and De La Haza, A. (2013). Condition assessment of concrete structures using a new analysis method: Ground-penetrating radar computer-assisted visual interpretation. Construction and Building Materials, Vol. 38, January, 1246-1254.

Tsai, D. M., \& Lin, C. T. (2003). Fast normalized cross correlation for defect detection. Pattern Recognition Letters, Elsevier, Vol. 24, Iss. 15, November, 2625-2631.

Tsai, D. M., Lin, C. T., \& Chen, J. F. (2003). The evaluation of normalized cross correlations for defect detection. Pattern Recognition Letters, Elsevier, Vol. 24, Iss. 15, November, 2525-2535. 